\title{
Congress Calendar
}

\section{Respirition}

\begin{tabular}{l}
\hline 23.2.-25.2.2012 \\
Strasbourg \\
France \\
\\
\hline 29.2.-2.3.2012 \\
Florence \\
Italy
\end{tabular}

\section{2.-3.3.2012}

Köln

Germany

\begin{tabular}{l} 
12.-16.3.2012 \\
Ancona \\
Italy \\
\hline
\end{tabular}

14.3.-17.3.2012

Dubai

UAE

17.3.2012

Kent

UK

23.3.-25.3.2012

Puerto de la Cruz

Tenerife, Spain

\section{3.-1.4.2012}

Nürnberg

Germany

30.3.-1.4.2012

Estoril

Portugal

\section{4.-24.4.2012}

New York, N.Y.

USA

\section{4.-27.4.2012}

Crans-Montana

Switzerland

\subsubsection{2}

Kent

UK

\begin{tabular}{l}
\hline 7.-11.5.2012 \\
Ancona \\
Italy
\end{tabular}

Lung Transplantation

AIPO 8th International Workshop

Respiratory Intensive Care Unit: Update in Respiratory Critical

Medicine

34. Jahrestagung der Gesellschaft für Pädiatrische Pneumologie

Weeks in Interventional Pulmonology Flexible and Rigid Bronchoscopy

\section{Gulf Thoracic Congress}

5th Thoracic Ultrasound Course for Chest Physicians

ICPP8 - International Course on Pediatric Pulmonology

53. Kongress der Deutschen Gesellschaft für Pneumologie und Beatmungsmedizin e.V. 2012

10th ERS Lung Science Conference

Rebuilding a Diseased Lung: Repair and Regeneration

V World Asthma \& COPD Forum

Annual Conference of the Swiss Respiratory Society (SRS),

Swiss Society of Pediatric Pneumology (SGPP),

Swiss Society for Thoracic Surgery (SGT) and

Swiss Underwater and Hyperbaric Medical Society (SUHMS)

Course on 'Safety in Chest Drain Insertions'

Weeks in Interventional Pulmonology Flexible and Rigid

Bronchoscopy

\section{Information:}

ERS Headquarters

Tel. +4121213 0101, Fax +41212130100

E-Mail school@ersnet.org

Internet www.ersnet.org/schoolcourses

Information:

AIPO Ricerche

E-Mail workshop2012@aiporicerche.it

Internet www.aiporicerche.it

\section{Information:}

www.paediatrische-pneumologie.com

\section{Information:}

Course Director: Prof. Stefano Gasparini, MD

E-Mail s.gasparini@fastnet.it, www.eabip.org

Organising Secretariat: AIPO Ricerche

Via Antonio da Recanate, 2, IT-20124 Milano (Italy)

Tel. +39023659 0354, Fax +390236590360

Dr. Carlo Zerbino

E-Mail direzionegenerale@aiporicerche.it

\section{Information:}

http://gulfthoracic.com/

\section{Information:}

www.thoracicultrasound.com

\section{Information:}

www.cipp-meeting.org

\section{Information:}

http://www.pneumologie.de/373.0.html

\section{Information:}

ERS Headquarters

Tel. +4121213 0101, Fax +41212130100

E-Mail school@ersnet.org

Internet www.ersnet.org/schoolcourses

\section{Information:}

Congress Secretariat

E-Mail info@wipocis.org

Internet www.wipocis.org

\section{Information:}

www.pneumo.ch

\section{Information:}

www.thoracicultrasound.com

\section{Information:}

Course Director: Prof. Stefano Gasparini, MD

E-Mail s.gasparini@fastnet.it, www.eabip.org

Organising Secretariat: AIPO Ricerche

Via Antonio da Recanate, 2, IT-20124 Milano (Italy)

Tel. +39023659 0354, Fax +3902 36590360

Dr. Carlo Zerbino

E-Mail direzionegenerale@aiporicerche.it 
18.5.-23.5.2012 San Francisco, Calif. USA

\section{5.-26.5.2012}

Bucharest

Romania

\section{5.-3.6.2012 \\ Barcelona}

Spain

\section{6.-18.6.2012 \\ Cleveland, Ohio}

USA

16.6.-20.6.2012
Geneva

Switzerland

\begin{tabular}{l}
\hline 25.-29.6.2012 \\
Ancona \\
Italy
\end{tabular}

30.6.-2.7.2012

Bangkok

Thailand

\begin{tabular}{l}
\hline 18.8.-21.8.2012 \\
Québec City \\
Canada \\
\hline 1.9.-5.9.2012 \\
Vienna \\
Austria \\
\hline
\end{tabular}

\subsection{0.-25.10.2012}

Atlanta, Ga.

USA

\begin{tabular}{l}
\hline 5.-9.11.2012 \\
Ancona \\
Italy \\
\end{tabular}

ATS 2012 - American Thoracic Society

Future meetings

ATS 2013, May 17-22, Philadelphia, Penn.

ATS 2014, May 16-21, San Diego, Calif.

ATS 2015, May 15-20, Denver, Colo.

ATS 2016, May 13-18, San Francisco, Calif.

ATS 2017, May 19-24, Washington, D.C.

TB and M/XDR-TB: From Clinical Management to Control and

Elimination

HERMES Summer School

17th World Congress for Bronchology and Interventional Pulmonology and 17th World Congress for Broncheosophagology

European Academy of Allergy and Clinical Immunology Congress 2012

Weeks in Interventional Pulmonology Flexible and Rigid

Bronchoscopy

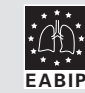

11th International Congress on Pediatric Pulmonology (CIPP XI)

XXI World Congress of Asthma

European Respiratory Society - Annual Congress

CHEST 2012

Future meeting

CHEST 2013, October 26-31, Chicago, III.

Weeks in Interventional Pulmonology Flexible and Rigid

Bronchoscopy
Information:

www.thoracic.org
Information:

ERS Headquarters

Tel. +4121213 0101, Fax +41212130100

E-Mail school@ersnet.org

Internet www.ersnet.org/schoolcourses

\section{Information:}

ERS Headquarters

Tel. +4121213 0101, Fax +41212130100

E-Mail school@ersnet.org

Internet www.ersnet.org/schoolcourses

Information: Atul C. Mehta, MD

President

17th WCBIP

E-Mail wcbip2012@gmail.com

\section{Information:}

www.eaaci2012.com/

\section{Information:}

Course Director: Prof. Stefano Gasparini, MD

E-Mail s.gasparini@fastnet.it, www.eabip.org

Organising Secretariat: AIPO Ricerche

Via Antonio da Recanate, 2, IT-20124 Milano (Italy)

Tel. +39023659 0354, Fax +390236590360

Dr. Carlo Zerbino

E-Mail direzionegenerale@aiporicerche.it

\section{Information:}

www.cipp-meeting.org

\section{Information:}

www.wca-2012.com

\section{Information:}

www.erscongress2012.org

Information: ACCP Member Services

3300 Dundee Road, Northbrook, IL 60062 (USA)

Tel. +1 847498 1400, Fax +1 8474985460

E-Mail registrationschestnet.org, www.chestnet.org

\section{Information:}

Course Director: Prof. Stefano Gasparini, MD

E-Mail s.gasparini@fastnet.it, www.eabip.org

Organising Secretariat: AIPO Ricerche

Via Antonio da Recanate, 2, IT-20124 Milano (Italy)

Tel. +39023659 0354, Fax +390236590360

Dr. Carlo Zerbino

E-Mail direzionegenerale@aiporicerche.it 